\title{
Gonadotropin-releasing hormone stimulation test and diagnostic cutoff in precocious puberty: a mini review
}

Siti Nadirah Ab Rahim, MBBS ${ }^{1,2}$, Julia Omar, MBBS, MPaTH, Tuan Salwani Tuan Ismail, MBBS, MPaTH ${ }^{2,4}$

${ }^{1}$ Faculty of Medicine \& Defence Health, National Defence University of Malaysia, Kuala Lumpur, Malaysia

2Department of Chemical Pathology, University Sains Malaysia, Kota Bharu, Malaysia

${ }^{3}$ Chemical Pathology Laboratory, University Sains Malaysia, Kota Bharu, Malaysia

${ }^{4}$ Hospital Universiti Sains Malaysia, Kota Bharu, Malaysia

Received: 30 December, 2019

Revised: 2 February, 2020

Accepted: 10 February, 2020

Address for correspondence:

Tuan Salwani Tuan Ismail, MBBS, MPaTH

Department of Chemical Pathology, School of Medical Sciences, University Sains Malaysia, 16150, Kubang Kerian, Kelantan, Malaysia

Tel: +60-136647129

Fax: $+60-97653370$

E-mail: tusti@usm.my

https://orcid.org/0000-0002-77398323
The gonadotropin-releasing hormone $(\mathrm{GnRH})$ stimulation test is a valuable tool in diagnosing and differentiating causes of early pubertal occurrences. Utility of the test can be limited in some instances, however, including the early phases of pubertal hypothalamic-pituitary-gonadal axis activation, in girls showing commonly overlapping pictures, and in obese children due to excess circulating estrogen that suppresses luteinizing hormone (LH). A lack of consistent baseline and stimulated gonadotropin cutoffs observed in different studies also contributes to limitations in testing. Nevertheless, early detection of true pathological causes for pubertal disorders is needed to allow prompt treatment and better prognosis. While basal $\mathrm{LH}$ can be beneficial as a good screening tool for detecting pubertal disorder, it does not preclude the need for $\mathrm{GnRH}$ testing. The aim of this review was to highlight the role of GnRH stimulation tests and varying testing cutoffs in diagnosis of precocious puberty and its classification.

Keywords: Gonadotropin-releasing hormone, Precocious puberty, GnRH analogues, Premature thelarche

\section{Introduction}

Gonadotropin-releasing hormone $(\mathrm{GnRH})$ stimulation tests assess activation of the hypothalamic-pituitary-gonadal axis (HPG axis). Administering GnRH analogues (GnRHa), together with determination of serum basal and poststimulated gonadotropin levels, predicts pubertal responses at pubertal ages. ${ }^{1,2)}$ One concern is the lack of agreement on gonadotropin cutoffs used to diagnose central precocious puberty (CPP). ${ }^{3-5)}$ The current review focuses on the clinical importance of GnRH testing in precocious puberty and different gonadotropin cutoffs utilized, along with limitations in testing.

\section{Diagnosis of precocious puberty}

In precocious puberty, differentiation of causes into 3 main categories is crucial. These categories are gonadotropin-dependent precocious puberty, gonadotropin-independent precocious puberty, and pubertal variants in patients with isolated signs of puberty without underlying pathology.

\section{Gonadotropin-dependent precocious puberty}

Challenges arise in distinguishing between true pathological causes of CPP, isolated premature thelarche in girls (nonprogressive variants), and early phases of normal puberty. ${ }^{3,6}$

Luteinizing hormone (LH) increments of $\geq 4-5$ IU/L at 30-60 minutes after GnRH 
administration are well-known criteria to diagnose CPP., ${ }^{1,7)}$ Values $>5 \mathrm{IU} / \mathrm{L}$ at both 40 and 45 minutes post-GnRH stimulation have shown $98 \%$ sensitivity and $100 \%$ specificity. ${ }^{8,9)}$ A peak value $>5 \mathrm{IU} / \mathrm{L}$ has been found in $88 \%$ of CPP patients at 1 hour post administration and in 100\% of CPP patients at 3 hours. ${ }^{10)}$ To further support diagnosis, a peak LH to follicle stimulating hormone $(\mathrm{LH} / \mathrm{FSH})$ ratio $>0.66$ has been added to the criteria, while a ratio $>0.45$ (sensitivity, $82 \%$; specificity, 97\%) has also been recognized. ${ }^{1,11)}$ Sensitivity and specificity for diagnosis, however, have been shown to be higher (100\%, respectively) with a ratio $>1.0$ at 1 hour and 3 hours in boys. ${ }^{10)}$ In contrast, in girls, the sensitivity of peak LH/FSH ratio $>1$ was only $50 \%{ }^{10)}$

Despite concerns over stimulated gonadotropin cutoff levels, basal LH has an equally crucial role. Levels $>1.0 \mathrm{IU} / \mathrm{L}$ have demonstrated a positive predictive value of $96.4 \%$-adequate to diagnose $\mathrm{CPP}$ - precluding the need to test stimulated gonadotropin levels. ${ }^{8)}$ Due to lower sensitivity (69.1\%) and specificity (50.5\%), however, basal LH levels $>1.1 \mathrm{IU} / \mathrm{L}$ are not recommended as a substitute to $\mathrm{GnRH}$ stimulation but rather as a means of screening in girls. ${ }^{12)}$ Caution must be exercised because as many as $35.7 \%$ of suspected cases have presented in girls with very low initial basal LH level who have later been diagnosed with $\mathrm{CPP}{ }^{13)}$ Some of these patients had basal LH levels lower than the analyzer detection limit. ${ }^{2)}$ Because LH levels $<0.2$ IU/L have $100 \%$ specificity and sensitivity in distinguishing between prepubertal and pubertal boys, a cutoff of $0.1 \mathrm{IU} / \mathrm{L}$ has been used. ${ }^{14)}$ Commonly, basal LH values $>0.3 \mathrm{IU} / \mathrm{L}$ and a stimulated level greater than $>5 \mathrm{IU} /$ $\mathrm{L}$ are distinctive in boys, but not in girls, especially at Tanner stages 2 and $3 .^{7,11)}$ Even for girls with low LH secretion (in early puberty), production of estradiol is sufficient to prompt pubertal changes. ${ }^{10)}$ Therefore, to avoid misdiagnosing CPP and overdiagnosing a true pubertal girl with CPP, further follow-up is required. Repeated GnRH tests can be performed in conjunction with other modalities, such as pelvic and abdominal ultrasound (to assess fundal/cervical ratio, uterus length, and ovarian volume), bone age assessment, and genetic studies. ${ }^{4,13)}$ Meanwhile, to minimize variation due to diurnal LH preferences in early phases of puberty, early-morning samples (before $10 \mathrm{AM}$ ) are desirable. ${ }^{15)}$ In addition, caution is needed in obese children as the peak response tends to be lower due to excess androgen and estrogen that suppress LH in obesity. ${ }^{16,17)}$ At Tanner stage 2, LH peaks have been shown to be significantly lower in obese and in overweight girls in comparison to normalweight girls $(9.1 \pm 6.1,10.4 \pm 6.4$, and $11.9 \pm 7.5 \mathrm{IU} / \mathrm{L}$, respectively). These differences have not been observed among stratified body mass index groups at Tanner stage $4 .{ }^{18-20)}$ A lower peak LH/FSH ratio of 0.6 has been seen in obese girls in comparison to 0.8 in normal-weight girls. ${ }^{18)}$

Efforts to differentiate CPP from early puberty using FSH level, especially in girls aged 7 to 8 years, have failed because basal and stimulated FSH level often is nonconclusive in these 2 categories at any point in various sampling periods. ${ }^{10,21)}$ Significantly higher basal FSH levels $>2.4$ IU/L have been found in CPP, but the increments shown by stimulated FSH level were indistinguishable from those in isolated premature thelarche. This ambiguity contributes to limitations in the use of the LH/FSH ratio in diagnosis of CPP in girls. ${ }^{4,5,12)}$ Prepubertal basal level of serum estradiol can reach down to $1 \mathrm{pg} / \mathrm{mL}$ and that of serum testosterone to $<10 \mathrm{ng} / \mathrm{dL}$, measurable with a highly sensitive method (i.e., tandem mass spectrometry). ${ }^{14)}$ Basal serum testosterone $>25 \mathrm{ng} / \mathrm{dL}$ to $>75 \mathrm{ng} / \mathrm{dL}$ in boys and estrogen level $>10 \mathrm{pg} / \mathrm{mL},>12 \mathrm{pg} / \mathrm{mL}$, or $>20 \mathrm{pg} / \mathrm{mL}$ in girls have been used as cutoff values for puberty. ${ }^{1,3,22,23)}$ With the GnRH test, peak values for both hormones are usually seen after 16 to 24 hours post-stimulation. ${ }^{1,3)}$ In girls, both LH response and peak LH/FSH ratio post-GnRH stimulation can sometimes be low up until midpuberty, indicating the usefulness of correlation with an estradiol peak up to $50 \mathrm{pg} / \mathrm{mL} 20-24$ hours after stimulation with leuprolide (a GnRHa) in identifying $\mathrm{CPP}^{23)}$

\section{Gonadotropin-independent precocious puberty}

High estradiol or testosterone level associated with blunted LH and FSH responses toward GnRH show a possibility of gonadotropin-independent precocious puberty. ${ }^{6)}$ Estradiol levels greater than $10 \mathrm{pg} / \mathrm{mL}$ are pubertal, but levels higher than $100 \mathrm{pg} / \mathrm{mL}$ suggest a gonadotropin-independent source, such as tumors or cysts. ${ }^{3,24)}$ In early puberty, testosterone level is higher in early-morning samples due to nighttime production of LH. Even in advanced stages of puberty, testosterone level may vary by the Tanner stage. Accordingly, diagnosis cannot be concluded with a single measurement.

\section{Pubertal variants}

In isolated premature thelarche, the results of post-GnRH stimulation tests can range from vivid prepubertal responses to increments of LH level mimicking pubertal responses. The LH/ FSH ratio in such variants, however, is often less than that in gonadotropin-dependent precocious puberty. ${ }^{14)}$ Distinguishing premature thelarche from CPP is crucial because, in the form of nonprogressive variants, premature thelarche does not require GnRHa therapy. ${ }^{6,14)}$ Pelvic ultrasound will show greater uterine and ovarian volumes in CPP, although findings can be indistinguishable from those of normal variants due to estrogen effects. ${ }^{4,14)}$ Ovarian volumes $\geq 1.3 \mathrm{~mL}$ and uterine lengths $\geq 30$ $\mathrm{mm}$, with a fundal cervical ratio $\geq 0.98$, are reliable predictors of CPP due to high sensitivity and specificity, especially when GnRH stimulation is not conclusive., ${ }^{4,10)}$

Though isolated premature menarche is extremely rare, cyclical bleeding in a prepubertal-aged girl should prompt performance of GnRH stimulation test only after exclusion of other more common causes such as trauma, infection, tumor, and sexual abuse. ${ }^{6}$

Investigation of premature adrenarche without breast development in girls or of testicular enlargement in boys should aim at the exclusion of excess androgen sources rather than activation of the HPG axis. As adrenal pathology, 
such as congenital adrenal hyperplasia $(\mathrm{CAH})$, should be first considered, baseline dehydroepiandrosterone sulfate, $17 \alpha$-hydroxyprogesterone, androstenedione, testosterone, and synacthen stimulation tests would be more relevant than the GnRH test. ${ }^{6}$ If GnRH tests are performed, however, gonadotropin values are expected to be prepubertal. In confirmed cases of $\mathrm{CAH}$, when there is a high suspicion of precocious puberty, the presence of gonadotropin response with pubertal changes suggests development of $\mathrm{CPP}$ in $\mathrm{CAH}^{3}{ }^{3,6223)}$

\section{Limitations of the GnRH stimulation test}

GnRH stimulation tests come with a number of limitations. In addition to a lack of standardized gonadotropin cutoffs, the timing of blood sampling and pharmacologic agents used also tend to vary. ${ }^{1)}$ Traditionally, GnRH has been administered via intravenous bolus at doses of $100 \mu \mathrm{g} / \mathrm{m}^{2}$ or $2.5 \mu \mathrm{g} / \mathrm{kg}$ with 5 to 8 subsequent blood samplings at 20 to 30 minutes. Modifications to the protocol were later made to improve practicality, including use of dosage infusion over 120 minutes or repetitive doses over a period of up to 36 hours, with fewer blood samples collected. ${ }^{25)}$ Today, GnRHa medications such as nafarelin, triptorelin, buserelin, and leuprolide are used instead. Studies reveal varying efficacy. ${ }^{3)}$ Accordingly, no single ideal analogue has been found to be superior to another. ${ }^{25)}$

Analytical issues in measurement assays are also a matter of concern in GnRH testing. Because, clinically speaking, LH level is clinically more implicated than that of FSH and reference intervals are age- and sex-specific, a detection limit of at least $0.1 \mathrm{IU} / \mathrm{L}$ is required for an assay. ${ }^{14)}$ Assays that are not sensitive enough at lower concentrations of hormones are prohibited for use in younger children. ${ }^{7)}$ Generally, radioimmunoassays have quite a high detection limit $(0.5$ to $2 \mathrm{IU} / \mathrm{L})$, followed closely by immunoradiometric assays $(0.1$ to $0.5 \mathrm{IU} / \mathrm{L})$. Both immunofluorometric assays and immunochemiluminescence assays are sensitive at $0.1 \mathrm{IU} / \mathrm{L} .{ }^{25}$ Although most current assays have overcome this limitation, worldwide standardization for measurement of gonadotropin is lacking, and assays are vulnerable to interferences such as heterophilic antibodies and crossreactive molecules, which reduce assay specificity. ${ }^{1,25,26)}$

\section{Conclusion}

GnRH stimulation tests have a diagnostic role in precocious puberty. To date, however, there are no consistent cutoffs for either pre- or poststimulated gonadotropin level. This is particularly challenging in girls due to marked variation in LH level, in obese children due to lower gonadotropin levels, and in most early presentation wherein diurnal variations in gonadotropin might affect results. Considering the limitations in GnRH testing, interpretation should be made in conjunction with clinical suspicions and supported by modalities, such as radiological imaging and growth velocity.

\section{Conflicts of interest}

No potential conflict of interest relevant to this article was reported.

\section{Acknowledgments}

For their valuable input, we would like to thank the Master's Students of the Chemical Pathology and Pediatric Departments of Hospital Universiti Sains Malaysia.

\section{References}

1. Kumar M, Mukhopadhyay S, Dutta D. Challenges and controversies in diagnosis and management of gonadotropin dependent precocious puberty: an Indian perspective. Indian J Endocrinol Metab 2015;19:228-35.

2. Mogensen SS, Aksglaede L, Mouritsen A, Sørensen K, Main KM, Gideon P, et al. Diagnostic work-up of 449 consecutive girls who were referred to be evaluated for precocious puberty. J Clin Endocrinol Metab 2011;96:1393-401.

3. Prasad HK, Khaldikar VV, Jahagirdar R, Khaldikar AV, Lalwani SK. Evaluation of GnRH analogue testing in diagnosis and management of children with pubertal disorders. Indian J Endocrinol Metab 2012;16:400-5.

4. Binay C, Simsek E, Bal C. The correlation between GnRH stimulation testing and obstetric ultrasonographic parameters in precocious puberty. J Pediatr Endocrinol Metab 2014;27:1193-9.

5. Nam HK, Rhie YJ, Son CS, Park SH, Lee KH. Factors to predict positive results of gonadotropin releasing hormone stimulation test in girls with suspected precocious puberty. J Korean Med Sci 2012;27:194-9.

6. Wei C, Davis N, Honour J, Crowne E. The investigation of children and adolescents with abnormalities of pubertal timing. Ann Clin Biochem 2016;54:20-32.

7. Menon PS. Precocious puberty, GnRH stimulation test and monitoring GnRH analog therapy. Indian J Pediatr 2015;82:980-2.

8. Kandemir N, Demirbilek H, Özön ZA, Gönç N, Alikaşifoğlu A. GnRH stimulation test in precocious puberty: single sample is adequate for diagnosis and dose adjustment. J Clin Res Pediatr Endocrinol 2011;3:12-7.

9. Kim HK, Kee SJ, Seo JY, Yang EM, Chae HJ, Kim CJ. Gonadotropin-releasing hormone stimulation test for precocious puberty. Korean J Lab Med 2011;31:244-9.

10. Yazdani P, Lin Y, Raman V, Haymond M. A single sample GnRHa stimulation test in the diagnosis of precocious puberty. Int J Pediatr Endocrinol 2012;2012:23.

11. Fuqua JS. Treatment and outcomes of precocious puberty: an update. J Clin Endocrinol Metab 2013;98:2198-207.

12. Lee HS, Park HK, Ko JH, Kim YJ, Hwang JS. Utility of basal luteinizing hormone levels for detecting central precocious puberty in girls. Horm Metabolic Res 2012;44:851-4.

13. Pasternak Y, Friger M, Loewenthal N, Haim A, Hershkovitz 
E. The utility of basal serum LH in prediction of central precocious puberty in girls. Eur J Endocrinol 2012;166:2959.

14. Carel JC, Eugster E, Rogol A, Ghizzoni L, Palmert MR. Consensus statement on the use of gonadotropin-releasing hormone analogs in children. Pediatrics 2009;123:e752-62.

15. Kang YS, Yoo DY, Chung IH, Yoo EG. Diurnal variation of gonadotropin levels in girls with early stages of puberty. Ann Pediatr Endocrinol Metab 2017;22:183-8.

16. Bordini B, Littlejohn E, Rosenfield RL. LH dynamics in overweight girls with premature adrenarche and slowly progressive sexual precocity. Int J Pediatr Endocrinol 2010;2010:724696.

17. Lee HS, Park HK, Ko JH, Kim YJ, Hwang JS. Impact of body mass index on luteinizing hormone secretion in gonadotropin-releasing hormone stimulation tests of boys experiencing precocious puberty. Neuroendocrinology 2013;97:225-31.

18. Fu JF, Liang JF, Zhou XL, Prasad HC, Jin JH, Dong GP, et al. Impact of BMI on gonadorelin-stimulated LH peak in premenarcheal girls with idiopathic central precocious puberty. Obesity 2015;23:637-43.

19. Heo S, Lee YS, Yu J. Basal serum luteinizing hormone value as the screening biomarker in female central precocious puberty. Ann Pediatr Endocrinol Metab 2019;24:164-71.
20. Lee HS, Yoon IS, Hwang JS. Luteinizing hormone secretion during gonadotropin-releasing hormone stimulation tests in obese girls with central precocious puberty. J Clin Res Pediatr Endocrinol 2016;8:392-8.

21. Kılıç A, Durmuş MS, Ünüvar E, Yıldız İ, Aydın BK, Uçar A, et al. Clinical and laboratory characteristics of children referred for early puberty: preponderance in 7-8 years of age. J Clin Res Pediatr Endocrinol 2012;4:208-12.

22. Karaoglan M, Keskin M, Kul S, Ozkur A. A diagnostic scoring system to distinguish precocious puberty from premature thelarche based on clinical and laboratory findings. Iran J Pediatr 2018;28:e64118.

23. Menon PS, Vijayakumar M. Precocious puberty-perspectives on diagnosis and management. Indian J Pediatr 2014;81:76-83.

24. Klein DA, Emerick JE, Sylvester JE, Vogt KS. Disorders of puberty: an approach to diagnosis and management. Am Fam Physician 2017;96:590-9.

25. Harrington J, Palmert MR. Distinguishing constitutional delay of growth and puberty from isolated hypogonadotropic hypogonadism: critical appraisal of available diagnostic tests. J Clin Endocrinol Metab 2012;97:3056-67.

26. Wheeler MJ. The measurement of LH, FSH, and prolactin. In: Wheeler M. editor. Hormone assays in biological fluids. methods in molecular biology (methods and protocols). Totowa (NJ): Humana Press, 2013:1065. 